

\title{
Robust Trajectory Tracking for a Flexible Probe in the Presence of Uncertainties and Disturbance
}

\author{
S. Zamiri' ${ }^{1}$ A. Vahidian Kamyad ${ }^{2}$ \\ ${ }^{1}$ Electrical Engineering Department, Gonabad Branch, Islamic Azad University, Gonabad, Iran \\ Zamiri.samira@yahoo.com \\ ${ }^{2}$ Electrical Engineering Department, Ferdowsi University of Mashhad. Mashhad, Iran \\ Kamyad@math.um.ac.ir
}

Article history:

Received July 2014

Accepted August 2014

Available online November 2014

\begin{abstract}
This paper investigates the trajectory tracking of a bio-inspired flexible probe in medical where there exist of uncertainty and disturbance in the system. In the first approach, a sliding mode controller is designed to deal with the uncertainties and output disturbances in the system. In this case, it is assumed that the upper band of uncertainty in the system is known, but in practice this may not be really possible. Therefore, in the next section, the sliding mode controller has been extended to a robust - adaptive controller in such a way that even if there is no information on the uncertainty upper bond, the system is still stable and the probe continues to track the desired trajectory. In this case, an adaptive rule has been designed to estimate the upper bound of the uncertainty and disturbance. A numerical simulation shows the effectiveness of the proposed methodologies.
\end{abstract}

Keywords: Bio-inspired flexible probe, Sliding mode control, Robust-adaptive control.

\section{Introduction}

Among a lot of techniques that are available for the surgeon, percutaneous intervention has attracted great interest, because it is performed through a very small port through the soft tissue, i.e., skin [1]. Entering into the body tissues through the skin and by probes and needles develops far fewer risks than open surgery for patients and thus is a treatment of choice in many clinical applications [2-3]. Needle insertion into soft tissue has many clinical applications such as tissue biopsy [4], brachytherapy [5], and anesthetic delivery [6]. When a path in to the tissue does not seem possible or is not safe, a flexible probe or needle can be utilized instead of a rigid probe to reach the target while avoiding special areas or obstacle avoidance. On the other hand, using flexible probe impose less pain to the patient.

Different models have been provided for bevel-tip flexible probe which predict needle-tip deviations from the target for compensation. Webster et al. proposed a kinematic model for probe deflections by generalizing the standard nonholonomic unicycle and bicycle models [7]. 
Due to the lack of an accurate on dynamic model of needle-tissue systems [8], most of the related studies are on computer simulations [9-10], pre-planning [11], steering [12] and path-planning [13], rather than the automatic control of the flexible needle. Path-planning, by itself, is not sufficient to proceed to robot-assisted needle insertion, unless there is a control system which can control the needle to track the designed path. Pre-planning is an offline process in which the needle insertion parameters such as the insertion angle and bevel orientation are derived prior to the insertion [11]. Steering is a closer approach to a control strategy. DiMaio et al. [12] derived a needle manipulation Jacobian, which relates the desired needle tip to base velocities, using a numerical needle insertion model that includes needle deflection and tissue deformation. Glozman et al. [14] used the analytical forward and inverse kinematics of a simplified needle-tissue model to determine the required needle base trajectory for any desired needle tip trajectory. Contrary to other approaches, we are currently developing a bio-inspired flexible probe [15-16], which can change its direction smoothly by means of a programmable bevel tip.

Robot-assisted needle insertion has recently received significant attention because of its potential capability in delivering accurate targeting [17], [18], [19], [20]. Due to the lack of an accurate model for the coupled needle-tissue system [21], most of the related studies are focused on computer simulations [22], [23], pre-planning [24], steering [25], and path-planning [26], rather than the automatic control of the flexible needle.

Closed loop control of the probe tip along an arbitrary smooth trajectory can be achieved without the need for an external imaging system. The authors in [27-28] have been showed that the concept of a programmable bevel tip is viable through experiments with a first $12 \mathrm{~mm}$ outer diameter prototype, where two-dimensional trajectory following can be achieved by means of a state feedback controller employing approximated linearization and a chained form representation. Webster et al. first proposed a kinematic model of the beveled tip needle to describe its behavior in soft tissue [29]. Alterovitz et al. then derived a motion-planning algorithm under uncertainty to obtain better targeting accuracy [30].

As the needle is inserted, asymmetric tissue forces cause the tip to deflect and follow a curved path through the tissue, which the flexible shaft follows. Asymmetric tip designs can be passive (e.g., using a beveled, prebent (kinked), precurved, or flexure tip) [31], [32] or actuatable [33], [34].

Reed et al. modeled the torsional dynamics of a flexible needle to analyze torsional behavior during needle insertion [35]. These studies have predominantly focused on open-loop trajectory following. Glozman and Shoham closed the control loop in their original approach [36] by means of fluoroscopic images, which were used to measure needle deflection during the insertion process [37]. Recently, [38] designed a model predictive controller for a bio-inspired flexible probe to track a desired trajectory. On the other hand, for generating multiple trajectories with a fixed bevel tip, Minhas et al. [39, 40] and Wood et al. [41] proposed a duty-cycling spinning algorithm - by periodically changing the orientation of the needle along its long axis by means of a base-mounted revolute actuator, trajectories with different radii could be achieved. Specifically, [42] utilized fluoroscopic images to measure the deflection of a stiff needle (without bevel tip) when inserted into tissue.

In previous studies on bio-inspired flexible probes, it has been assumed that the model of probe is exact and there is no disturbance and uncertainty in the system. Actually, according to the movement of the probe tip into the tissue and the lack of detailed knowledge of the tissue, the model is subject to the noise and disturbance. This unknown disturbance may accrue because of the vessels and the unpredictable movement of tissues in the direction of the probe path. In this case, a term as disturbance and noise must be added to the kinematic model of probe.

In this paper, the problem of trajectory tracking of a bio-inspired flexible probe with consideration of uncertainty and disturbance in the system model has been studied. With assumption that the upper band of uncertainty in the system is known a sliding mode controller is designed to deal with the 
uncertainties and output disturbances in the system. In practice, knowing the upper bound of disturbances may not be really possible. Therefore, the sliding mode controller has been extended to a robust-adaptive controller in such a way that, even if there is no information on the uncertainty upper bond, the probe continues to track the desired trajectory.

This paper is organized as follows: The problem formulation and some preliminaries are given in Section II. Section III presents the main results of this paper that is designing the tracking controller for the probe in presence of uncertainty and disturbance. A robust sliding mode controller and a robust- adaptive controller have been designed in this Section. Simulation results in Section IV are given to confirm the analytical results. Finally, conclusions in Section V close the paper.

\section{Problem formulation}

The kinematic model of a bio-inspired flexible probe can be expressed by the following nonlinear model [24]:

$$
\left[\begin{array}{c}
\dot{x} \\
\dot{y} \\
\dot{\theta} \\
\dot{\delta}
\end{array}\right]=\left[\begin{array}{c}
\cos \theta \\
\sin \theta \\
k(\delta-\varepsilon \theta) \\
0
\end{array}\right] u_{1}+\left[\begin{array}{l}
0 \\
0 \\
0 \\
1
\end{array}\right] u_{2}
$$

where $\delta$ indicates the steering offset, $\mathrm{k}$ indicates a coefficient between the steering offset and the curvature of the probe, $\varepsilon$ indicates the compensation coefficient caused by the shape of the probe, $\theta$ indicates the tip angle of the probe and $\mathrm{u}_{1}$ and $\mathrm{u}_{2}$ respectively denote the linear velocity and the rate of change of steering offset of the probe (Fig. 1)

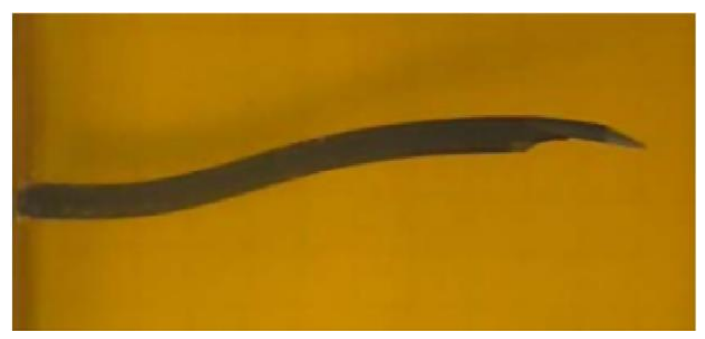

(a)

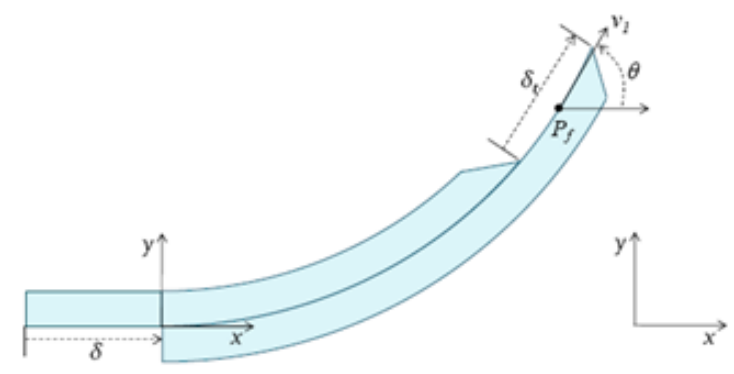

(b)

Fig. 1 The bio-inspired flexible probe. The probe that is following a trajectory [24]. The model of a Flexible probe 
Actually, the bevel tip of probe is controlled by applying a force to the base of the probe and probe's motion is due to the relative position between two segments of the probe so-called steering offset [18]. The aim of the probe control is leading to an optimal point in the tissue of the body. To reach this point, an optimal path is determined to deal with possible obstacles or damaging to the body tissues.

Defining $\mathrm{q}=[\mathrm{x}, \mathrm{y}, \theta, \delta]$ as the generalized coordinates of the probe and $\mathrm{u}=\left[\mathrm{u}_{1}, \mathrm{u}_{2}\right]^{\mathrm{T}}$ as input velocities, the model in (1) can be written as

$\dot{q}=G(q) u$

Where

$G(q)=\left[\begin{array}{cc}\cos \theta & 0 \\ \sin \theta & 0 \\ \mathrm{k}(\delta-\varepsilon \theta) & 0 \\ 0 & 1\end{array}\right]$

The model introduced in (2) does not express the exact behavior of the probe. For example, the relation between instantaneous curvature $(\rho)$ and the steering offset in the tip of probe $\left(\delta_{t}\right)$ has been considered with a linear function with the coefficient $\mathrm{k}\left(\mathrm{mm}^{-2}\right)$ [24]. This relation may be more complicated and with this assumption, some parts of the motion behavior of probe may be ignored. So, in the following model, we have considered such modeling uncertainties

$\dot{q}=(G(q)+\Delta G(q)) u$

Where $\Delta \mathrm{G}(\mathrm{q})$ denotes the additive uncertainty in the kinematic model introduced in (2).

In addition, according to the movement of the probe tip into the tissue and the lack of detailed knowledge of the tissue, the model in (2) is subject to the noise and disturbance. This unknown disturbance may accrue because of the vessels and the unpredictable movement of tissues in the direction of the probe path. In this case, a term as disturbance and noise must be added to the kinematic model in (2). In fact, considering the effects of noise and disturbance, more accurately describes the behavior of the probe. So, rewrite the model in (2) as

$\dot{q}=G(q) u+E(t)$

Where $\mathrm{E}(\mathrm{t})=\left[\mathrm{E}_{1}(\mathrm{t}), \mathrm{E}_{2}(\mathrm{t}), \mathrm{E}_{3}(\mathrm{t}), \mathrm{E}_{4}(\mathrm{t})\right]^{\mathrm{T}}$ denotes the disturbance and noise in the probe model that there is no information about them, unless we assume that this disturbance is bounded and we know the upper bound of it i.e.

$\left|E_{i}(t)\right| \leq \alpha_{i} i=1, \ldots, 4$

where $\alpha_{i} \quad i=1, \ldots, 4$ indicate the upper bound of disturbance and noise.

Considering both uncertainty and disturbance in the kinematic model of probe, one can easily combine the two models in (4) and (5) as follow

$\dot{q}=G(q) u+d(t)$

$$
d(t)=\Delta G(q) u+E(t)
$$


In this model $d(t)=\left[d_{1}(t), d_{2}(t), d_{3}(t), d_{4}(t)\right]^{T}$ describes both the model uncertainty and disturbance in the system. It is assumed that we only know the upper bound of the elements ofd(t)as follows

$\left|d_{i}(t)\right| \leq \varepsilon_{i} i=1, \ldots, 4$

Where $\varepsilon_{\mathrm{i}} \in \mathrm{R}^{+}$denotes the upper bound of the summation of the disturbance and uncertainty in (7).

Now, according to the (2) and (7), the equation of each state variable can be written as follow

$$
\begin{aligned}
& \dot{x}=\cos \cos (\theta) u_{1}+d_{1} \quad\left|d_{1}(t)\right| \leq \varepsilon_{1} \\
& \dot{y}=\sin \sin (\theta) u_{1}+d_{2} \quad\left|d_{2}(t)\right| \leq \varepsilon_{2} \\
& \dot{\theta}=k(\delta-\varepsilon \theta) u_{1}+d_{3}\left|d_{3}(t)\right| \leq \varepsilon_{3} \\
& \dot{\delta}=u_{2}+d_{4}\left|d_{4}(t)\right| \leq \varepsilon_{4}
\end{aligned}
$$

\section{Design of tracking controller}

The aim of this Section is to design a controller for the flexible probe that is described in (9-12), to track a desired trajectory. Note that the rank of the matrix G(q) in (3) around any equilibrium point is two. This means that only two state variable of the probe in the model can be controlled to reach a desired point or track a desired trajectory. In this Section, a sliding mode controller has been designed in such a way that the position of the probe tip e.g. $[\mathrm{x}, \mathrm{y}]^{\mathrm{T}}$ tracks a desiredtrajectory $\left[\mathrm{x}_{\mathrm{des}}, \mathrm{y}_{\mathrm{des}}\right]^{\mathrm{T}}$.

In the part A of this Section, a sliding mode controller is designed for the system in (9-12) to deal with the uncertainties and disturbances. In this case, it is assumed that the upper band of uncertainty in the system is known, but we know that in practice, this may not be really possible. Therefore, in part B, a robust - adaptive controller has been designed in such a way that, even if there is no information on the uncertainty upper bond, the system is still stable and the probe continues to track the desired trajectory.

\section{A. Robust sliding mode controller}

The sliding mode controller has been designed using back stepping in the following three steps:

Step 1: design $\mathrm{u}_{1}$ and $\theta$ in (9) and (10) in such a way that $[\mathrm{x}, \mathrm{y}]^{\mathrm{T}} \operatorname{tracks}\left[\mathrm{x}_{\mathrm{des}}, \mathrm{y}_{\mathrm{des}}\right]^{\mathrm{T}}$.

Step 2: design $\delta$ in (11) in such a way that $\theta$ converges to $\theta$ that is designed in the previous step $\left(\theta_{\text {des }}\right)$.

Step 3: design $\mathrm{u}_{2}$ in (12) in such a way that $\delta$ converges to $\delta$ that is designed in the previous step $\left(\delta_{\text {des }}\right)$.

\section{Step 1: Design $u_{1}$ and $\theta$}

First, consider the following change of variables

$$
v_{1}=\cos \cos (\theta) u_{1}
$$


$v_{2}=\sin \sin (\theta) u_{1}$

So, the equations in (9) and (10) can be written as

$\dot{x}=v_{1}+d_{1}$

$\dot{y}=v_{2}+d_{2}$

Now, the problem is finding $v_{1}$ and $v_{2}$ in presence of unknown uncertainties $d_{1}$ and $d_{2}$ such thatx and $\mathrm{y}$ converge to the $\mathrm{x}_{\mathrm{des}}$ and $\mathrm{y}_{\mathrm{des}}$ respectively. The controller design has been presented for the first equation in (14) and designing controller for the second equation in (14) is quite similar.

Considering the error as $\tilde{\mathrm{x}}=\mathrm{x}-\mathrm{x}_{\mathrm{des}}$, we choose the integral sliding surface $\mathrm{s}(\mathrm{t})$ as below

$s(t)=\tilde{x}+\int^{t} \Lambda \tilde{x}(\tau) d \tau$

0

Where $\mathrm{s}(\mathrm{t}) \in \mathbb{R}$ and $\Lambda>0$ is an arbitrary positive constant.

To establish the sliding mode, the reaching condition has been selected as

$$
\dot{s}(t)=-r s-\rho \operatorname{sgn}(s)
$$

Where $r$ and $\rho$ are positive constants.

We select the input control $v_{1}$ as follow for the system in (14)

$v_{1}=\dot{x}_{d e s}-\Lambda \tilde{\mathrm{x}}-r s-\rho \operatorname{sgn}(s)$

Substituting (17) into (14) results in the following equation for the closed loop system

$$
\dot{s}(t)=-r s-\rho \operatorname{sgn}(s)+d_{1}
$$

It is shown in the following theorem that the input control (17) will stabilize the system in (14) if $\rho>$ $\varepsilon_{1}$. This means that we should know the upper bound ofd $\mathrm{d}_{1}$.

Theorem1. Consider the first equation in (14). By applying the input control (17) with $\Lambda>0, r>0$ and $\rho>\varepsilon_{1}$, the closed loop system of error is asymptotically stable and $\mathrm{x}$ exponentially converges to $\mathrm{x}_{\mathrm{des}}$. In this case, $\varepsilon_{1}$ denotes the upper bound of the uncertainty and disturbance denoted by $\mathrm{d}_{1}$.

Proof: Consider the following Lyapunov candidate function:

$$
V=\frac{1}{2} s^{2}
$$

Taking the derivative of $\mathrm{V}$ with respect to time and substituting from (14), (15) and (17) results in

$$
\begin{aligned}
& \dot{V}=s \dot{s}=s(\dot{\tilde{x}}+\Lambda \tilde{x})=s(\dot{x}-\dot{x} d e s+\Lambda \tilde{x})=s\left(v_{1}+d_{1}-\dot{x}_{d e s}+\Lambda \bar{x}\right)=s\left(\dot{x}_{d e s}-\Lambda \tilde{x}-r s-\rho \operatorname{sgn}(s)+d_{1}-\dot{x}_{d e s}+\Lambda \tilde{x}\right)=s\left(-r s-\rho \operatorname{sgn}(s)+d_{1}\right) \\
& =s\left(-r s-\rho \operatorname{sgn}(s)+d_{1}\right)=-r|s|^{2}-\rho|s|+s d_{1} \leq-r|s|^{2}-\rho|s|+|s|\left|d_{1}\right| \leq-r|s|^{2}+\left(\varepsilon_{1}-\rho\right)|s|
\end{aligned}
$$


Equation (20) shows that the condition $\rho>\varepsilon_{1}$ is a sufficient condition for the derivative of lyapunov function to be negative.

Using Lyapunov theorems, negativity of the derivative of Lyapunov function shows that $s(t)$ and $\dot{\mathrm{s}}(\mathrm{t})$ tends to zero. So

$$
\dot{s}(t)=\dot{\tilde{x}}+\Lambda \tilde{x}=0 \Rightarrow \dot{\tilde{x}}=-\Lambda \tilde{x}
$$

And this means that

$$
\dot{\tilde{x}}=-\Lambda \tilde{x} \Rightarrow \tilde{x}=e^{-\Lambda t} \tilde{x}(0) \Rightarrow \tilde{x} \rightarrow 0 \Rightarrow x \rightarrow x \text { des }
$$

Design of the input controller $v_{2}$ for the second equation in (14) is similar to (17). So, without loss of generality we can consider both $\mathrm{v}_{1}$ and $\mathrm{v}_{2}$ as follow

$$
\begin{aligned}
& v_{1}=\dot{x}_{d e s}-\Lambda_{1} \tilde{x}-r_{1} s_{1}-\rho_{1} \operatorname{sgn}\left(s_{1}\right) \\
& v_{2}=\dot{y}_{d e s}-\Lambda_{2} \tilde{y}-r_{2} s_{2}-\rho_{2} \operatorname{sgn}\left(s_{2}\right)
\end{aligned}
$$

Where $\Lambda_{1}, \Lambda_{2}, r_{1}, r_{2}$ are real positive constants and the inequalities $\rho_{1}>\varepsilon_{1}$ and $\rho_{2}>\varepsilon_{2}$ should be satisfied. On the other hand, the sliding surfaces $s_{1}$ and $s_{2}$ are defined as follow

$s_{1}(t)=\tilde{x}+\int_{0}^{t} \Lambda_{1} \tilde{x}(\tau) d \tau$

$s_{2}(t)=\tilde{y}+\int_{0}^{t} \Lambda_{2} \tilde{y}(\tau) d \tau$

Now, after achieving $\mathrm{v}_{1}$ and $\mathrm{v}_{2}$ from (23), according to the equations in (13) $\mathrm{u}_{1}$ and the desired $\theta$ $\left(\theta_{\text {des }}\right)$ can be obtained from the following relations

$$
\begin{aligned}
& u_{1}=\sqrt{v_{1}^{2}+v_{2}^{2}} \\
& \theta_{d e s}=\tan ^{-1}\left(\frac{v_{2}}{v_{1}}\right)
\end{aligned}
$$

Assumption 1: It is assumed in this paper thatu $u_{1} \neq 0$.

This assumption means that during achieving the desired point, the probe must not be stopped.

\section{Step 2: Design $\delta$}

In this step, the steering offset $\delta$ in (11) has been designed in such a way that $\theta$ converges to $\theta_{\text {des }}$ in (25).

Consider the equation in (11) as follows 
$\dot{\theta}=k(\delta-\varepsilon \theta) u_{1}=A \theta+B \delta+d_{3}$



$s_{3}(t)=\tilde{\theta}+\int_{0}^{t} \Lambda_{3} \tilde{\theta}(\tau) d \tau$

where $\widetilde{\theta}=\theta-\theta_{\text {des }}$ and $\Lambda_{3}$ is a arbitrary positive constant. Now, choosing $\delta$ as below for the system in (26) results in $\theta \rightarrow \theta_{\text {des }}$

$\delta_{d e s}=\frac{1}{B}\left\{\dot{\theta}_{d e s}-\Lambda_{3} \tilde{\theta}-A \theta-r_{3} s_{3}-\rho_{3} \operatorname{sgn}\left(s_{3}\right)\right\}$

Where $r_{3}, \Lambda_{3}$ and $\rho_{3}$ are positive constants. It should be noted that according to the Assumption $1, \mathrm{~B} \neq$ 0 and we can use $1 / \mathrm{B}$ in (26).

Theorem2. Consider the equation in (11). By applying the input steering offset $\delta$ as in (28) with $\Lambda_{3}>$ $0, r_{3}>0$ and $\rho_{3}>\varepsilon_{3}$, the closed loop system of error is asymptotically stable and $\theta$ exponentially converges to $\theta_{\text {des }}$. In this case $\varepsilon_{3}$ denotes the upper bound of the uncertainty and disturbanced ${ }_{3}$.

Proof: consider the following lyapunov candidate function:

$$
V=\frac{1}{2} s_{3}^{2}
$$

Taking the derivative of $\mathrm{V}$ with respect to time results in

$$
\begin{aligned}
\dot{V}=s_{3} \dot{s}_{3}=s_{3}(\dot{\tilde{\theta}} & \left.+\Lambda_{3} \tilde{\theta}\right)=s_{3}\left(\dot{\theta}-\dot{\theta}_{d e s}+\Lambda_{3} \tilde{\theta}\right)=s_{3}\left(A \theta+B \delta+d_{3}-\dot{\theta}_{\text {des }}+\Lambda_{3} \tilde{\theta}\right) \\
& =s_{3}\left(A \theta+B \frac{1}{B}\left\{\dot{\theta}_{d e s}-\Lambda_{3} \tilde{\theta}-A \theta-r_{3} s_{3}-\rho_{3} \operatorname{sgn}\left(s_{3}\right)\right\}+d_{3}-\dot{\theta}_{d e s}+\Lambda_{3} \tilde{\theta}\right) \\
& =s_{3}\left(-r_{3} s-\rho_{3} \operatorname{sgn}\left(s_{3}\right)+d_{3}\right)=-r_{3}\left|s_{3}\right|^{2}-\rho_{3}\left|s_{3}\right|+s_{3} d_{3} \\
& \leq-r_{3}\left|s_{3}\right|^{2}-\rho_{3}\left|s_{3}\right|+\left|s_{3}\right|\left|d_{3}\right| \leq-r_{3}\left|s_{3}\right|^{2}+\left(\varepsilon_{3}-\rho_{3}\right)\left|s_{3}\right|
\end{aligned}
$$

Equation (30) shows that the condition $\rho_{3}>\varepsilon_{3}$ is a sufficient condition for the negativity of derivative of lyapunov function.

Similar to the Theorem 1 and using lyapunov conditions, negativity of the derivative of lyapunov function results in convergence of $\theta$ to $\theta_{\mathrm{des}}$.

\section{Step 3: Design $\mathbf{u}_{2}$}

The last step of designing the tracking controller is to design $\mathrm{u}_{2}$ in (12) such that $\delta$ converges to the $\delta_{\text {des }}$ in (28). This $u_{2}$ has been offered as below

$$
u_{2}=\dot{\delta}_{d e s}-\Lambda_{4} \tilde{\delta}-r_{4} s_{4}-\rho_{4} \operatorname{sgn}\left(s_{4}\right)
$$

Where 


$$
s_{3}(t)=\tilde{\delta}+\int_{0}^{t} \Lambda_{4} \tilde{\delta}(\tau) d \tau \quad \Lambda_{4}>0 \quad \mathrm{r}_{4}>0 \quad \rho_{4}>\varepsilon_{4}
$$

Theorem3. Consider the equation in (12). By applying $\mathrm{u}_{2}$ as (31) with $\Lambda_{4}>0, \mathrm{r}_{4}>0$ and $\rho_{4}>\varepsilon_{4}$, the closed loop system is asymptotically stable and $\delta$ exponentially converges to $\delta_{\text {des }}$. In this case $\varepsilon_{4}$ denotes the upper bound of the uncertainty and disturbanced . $_{\text {. }}$

Proof. The proof is similar to the proof of theorems 1 and 2.

By designing $\mathrm{u}_{1}$ and $\mathrm{u}_{2}$ from Theorems 1 and 3 respectively, the probe tip tracks the desired path $\left[\mathrm{x}_{\mathrm{des}}, \mathrm{y}_{\mathrm{des}}\right]^{\mathrm{T}}$ in the presence of uncertainty and disturbanced $(\mathrm{t})$.

\section{B. Robust-Adaptive controller}

In the previous part, it has been assumed that the upper band of uncertainties in the system e.i. $\varepsilon_{i}, i=$ $1, \ldots, 4$ are known and available, while it may not be possible in practice. Therefore, in this part, the previous controllers have been extended and for each equation, a robust- adaptive controller has been designed in such a way that the system tracks the desired trajectory even if there is no information of the upper band of uncertainty and disturbance in the system. For each case, a sliding mode control has been used and an adaptive law has been designed to estimate the upper bound of the uncertainty.

Similar to the part A, the robust-adaptive tracking controller is designed in three steps:

\section{Step 1: Design $u_{1}$ and $\theta$}

Consider the first equation in (14) and suppose that $\bar{\varepsilon}_{1}$ is an estimation of the upper bound $\operatorname{ofd}_{1}\left(\varepsilon_{1}\right)$. Now, instead of (17), consider the input control $v_{1}$ as follows

$$
v_{1}=\dot{x}_{d e s}-\Lambda \tilde{x}-r s-\rho \bar{\varepsilon}_{1} \operatorname{sgn}(s)
$$

Where the parameter $\bar{\varepsilon}_{1}$ is obtained from the following adaptive update law

$$
\dot{\bar{\varepsilon}_{1}}=K \rho \operatorname{ssgn}(s)
$$

Where $\mathrm{K}>0$ is adaptive gain? Actually $\bar{\varepsilon}_{1}$ that is an estimation of upper bound of uncertainty and disturbance, is estimated from the equation in (34). Now, consider the following Theorem.

Theorem4. Consider the first equation in (14). By applying the input control (33) with $\Lambda>0, r>0$ and $\rho>1$ along with the adaptive law in (34) withK $>0$, theclosed loop system of error is asymptotically stable and $\mathrm{x}$ exponentially converges to $\mathrm{x}_{\mathrm{des}}$.

Proof: Consider the following lyapunov candidate function:

$$
V=\frac{1}{2} s^{2}+\frac{1}{2 k} \tilde{\varepsilon}_{1}(t)^{2}
$$

Where $\tilde{\varepsilon}_{1}(\mathrm{t})=\bar{\varepsilon}_{1}(\mathrm{t})-\varepsilon_{1}$. Taking the derivative of $\mathrm{V}$ with respect to time and substituting from (33) and (34) results in 


$$
\begin{aligned}
\dot{V}=s \dot{s}+\frac{1}{k} \tilde{\varepsilon}_{1} \dot{\varepsilon}_{1} & =s\left(-r s-\rho \overline{\varepsilon_{1}} \operatorname{sgn}(s)+d_{1}\right)+\frac{1}{k} \tilde{\varepsilon}_{1} \dot{\bar{\varepsilon}}_{1} \\
& =-r|s|^{2}-\rho \bar{\varepsilon}_{1} \operatorname{ssgn}(s)+s d_{1}+\frac{1}{k} \tilde{\varepsilon}_{1} \operatorname{ses} \operatorname{sgn}(s) \\
& =-r|s|^{2}-\rho \bar{\varepsilon}_{1} \operatorname{ssgn}(s)+s d_{1}+\left(\bar{\varepsilon}_{1}-\varepsilon_{1}\right) \rho s \operatorname{sgn}(s) \\
& \leq-r|s|^{2}-\varepsilon_{1} \rho s \operatorname{sgn}(s)+|s| \varepsilon_{1} \leq-r|s|^{2}+(1-\rho) \varepsilon_{1}|s|<0
\end{aligned}
$$

Equation (36) shows that the condition $\rho>1$ is a sufficient condition for the derivative of lyapunov function to be negative. Similar to the Theorem 1, negativity of the derivative of lyapunov function results in convergence of $x$ tox $x_{\text {des }}$.

As it is clear, design of the input controller $v_{2}$ for the second equation in (14) is similar to (33). So, without loss of generality we can consider both $v_{1}$ and $v_{2}$ as follow

For the first equation in (14) we have

$$
\begin{aligned}
& v_{1}=\dot{x}_{d e s}-\Lambda_{1} \tilde{x}-r_{1} s_{1}-\rho_{1} \overline{\varepsilon_{1}} \operatorname{sgn}\left(s_{1}\right) \\
& \dot{\bar{\varepsilon}}_{1}=K_{1} \rho_{1} s_{1} \operatorname{sgn}\left(s_{1}\right)
\end{aligned}
$$

And for the second equation we have

$$
\begin{aligned}
& v_{2}=\dot{y}_{d e s}-\Lambda_{2} \tilde{y}-r_{2} s_{2}-\rho_{2} \bar{\varepsilon}_{2} \operatorname{sgn}\left(s_{2}\right) \\
& \dot{\bar{\varepsilon}}_{2}=K_{2} \rho_{2} s_{2} \operatorname{sgn}\left(s_{2}\right)
\end{aligned}
$$

Where $\Lambda_{1}, \Lambda_{2}, r_{1}, r_{2}, K_{1}$ and $K_{2}$ are real positive constants and the conditions $\rho_{1}>1$ and $\rho_{2}>1$ are satisfied. After designing $\mathrm{v}_{1}$ and $\mathrm{v}_{2}, \mathrm{u}_{1}$ and $\theta_{\mathrm{des}}$ can be obtained from the equation in (25).

\section{Step 2: Design $\delta$}

The steering offset $\delta$ in (11) has been designed in this step, in such a way that even if there is no information ond ${ }_{3}, \theta$ converges to $\theta_{\text {des }}\left(\theta_{\text {des }}\right.$ is obtained from the previous step)

Consider the equations in (26) and (27), choose $\delta$ as below for the system in (26)

$\delta_{d e s}=\frac{1}{B}\left\{\dot{\theta}_{\text {des }}-\Lambda_{3} \tilde{\theta}-A \theta-r_{3} s_{3}-\rho_{3} \bar{\varepsilon}_{3} \operatorname{sgn}\left(s_{3}\right)\right\}$

Where $r_{3}, \Lambda_{3}$ and $\rho_{3}$ are positive constants and $\bar{\varepsilon}_{3}$ is obtained from the following adaptive law

$\dot{\bar{\varepsilon}}=K_{3} \rho_{3} s_{3} \operatorname{sgn}\left(s_{3}\right)$

Where $\mathrm{K}_{3}>0$ is the adaptive gain?

Theorem5. Consider the equation in (11). By applying the input steering offset $\delta$ as (39) with $\Lambda_{3}>0$, $r_{3}>0$ and $\rho_{3}>1$ along with the adaptive law in (40) with $K_{3}>0, \theta$ exponentially converges to $\theta_{\text {des }}$. In this case $\bar{\varepsilon}_{3}$ denotes the estimation of the upper bound of the uncertainty and disturbanced ${ }_{3}$.

Proof: consider the following lyapunov candidate function:

$V=\frac{1}{2} s_{3}^{2}+\frac{1}{2 k_{3}} \tilde{\varepsilon}_{3}(t)^{2}$

Taking the derivative of $\mathrm{V}$ with respect to time results in 


$$
\begin{aligned}
\dot{V}=s_{3} \dot{s}_{3}+\frac{1}{k_{3}} \tilde{\varepsilon}_{3} \dot{\tilde{\varepsilon}}_{3} & =s_{3}\left(-r_{3} s_{3}-\rho_{3} \bar{\varepsilon}_{3} \operatorname{sgn}\left(s_{3}\right)+d_{3}\right)+\frac{1}{k_{3}} \tilde{\varepsilon}_{3} \dot{\varepsilon}_{3} \\
& =-r_{3}\left|s_{3}\right|^{2}-\rho_{3} \bar{\varepsilon}_{3} s_{3} \operatorname{sgn}\left(s_{3}\right)+s_{3} d_{3}+\left(\overline{\varepsilon_{3}}-\varepsilon_{3}\right) \rho_{3} s_{3} \operatorname{sgn}\left(s_{3}\right) \\
& \leq-r_{3}\left|s_{3}\right|^{2}-\varepsilon_{3} \rho_{3} s_{3} \operatorname{sgn}\left(s_{3}\right)+\varepsilon_{3}\left|s_{3}\right| \leq-r_{3}\left|s_{3}\right|^{2}+\left(1-\rho_{3}\right) \varepsilon_{3}\left|s_{3}\right|<0
\end{aligned}
$$

Equation (42) shows that the condition $\rho_{3}>1$ is a sufficient condition for the negativity of derivative of lyapunov function.

Similar to the Theorem 4 and using lyapunov conditions, negativity of the derivative of lyapunov function results in convergence of $\theta$ to $\theta_{\mathrm{des}}$.

\section{Step 3: Design $u_{2}$}

The last step of designing the tracking controller is to design $\mathrm{u}_{2}$ in (12) such that $\delta$ converge to the $\delta_{\text {des }}$ in (39). This $\mathrm{u}_{2}$ has been offered as below

$u_{2}=\dot{\delta}_{\text {des }}-\Lambda_{4} \tilde{\delta}-r_{4} s_{4}-\rho_{4} \bar{\varepsilon}_{4} \operatorname{sgn}\left(s_{4}\right)$

Where $\bar{\varepsilon}_{4}$ is an estimation of $\varepsilon_{4}$ and is obtained from the following equation

$$
\dot{\bar{\varepsilon}}_{4}=K_{4} \rho_{4} s_{4} \operatorname{sgn}\left(s_{4}\right), \quad K_{4}>0
$$

Theorem6. Consider the equation in (12). By applying $u_{2}$ in (43) with $\Lambda_{4}>0, r_{4}>0$ and $\rho_{4}>1$, the closed loop system of error is asymptotically stable and $\delta$ exponentially converges to $\delta_{\text {des }}$. In this case $\bar{\varepsilon}_{4}$ denotes the estimation of the upper bound of the uncertainty and disturbanced ${ }_{4}$.

Proof. The proof is similar to the proof of Theorems 4 and 5.

By designing $\mathrm{u}_{1}$ and $\mathrm{u}_{2}$ from Theorems 4 and 6 respectively, the probe tip tracks the desired path $\left[\mathrm{x}_{\mathrm{des}}, \mathrm{y}_{\mathrm{des}}\right]^{\mathrm{T}}$ in the presence of uncertainty and disturbance $\mathrm{d}(\mathrm{t})$ and when there is no information ond $(\mathrm{t})$.

\section{Numerical simulations}

Some numerical examples have been presented in this Section to clarify the results of the previous section

Example 1: Consider the following desired path for the flexible probe

$x_{\text {des }}=t$
$y_{\text {des }}=\sin \left(\frac{t}{2}\right)$

Suppose the uncertainty and disturbance $d_{i}(t), i=1,2, \ldots, 4$ for all equations in (9)-(12) is a random number between 1 and 2. So, an upper bound for $\mathrm{d}_{\mathrm{i}}(\mathrm{t})$ can be considered as $\left(\varepsilon_{1}=\varepsilon_{2}=\varepsilon_{3}=\varepsilon_{4}=2\right)$. The aim is to design an robust sliding mode controller using theorems 1-4. To use this theorem we choose $\rho=$ 3 for all of them that is larger than the upper bound of uncertainties. The other controller parameters have been chosen as follow (they have been considered same for all of sliding mode controllers)

$\Lambda=5, \quad \mathrm{r}=3$

On the other hand, the probe parameters have been considered as [24]

$\varepsilon=7.2, \quad \mathrm{k}=0.28$

Considering the initial condition of probe as $\left[\mathrm{x}_{0}, \mathrm{y}_{0}\right]^{\mathrm{T}}=[1,-2]^{\mathrm{T}}$, the results of simulation have been shown in Fig. 1 and Fig. 2. The tracking errors have been shown in Fig. 1. This figure confirms that errors converge to zero. It is worth mention that some oscillations in the error signals that called 
chattering, are because of the function sgn(s) in the input controllers. To counteract this phenomenon, there are some ways for example adding a damped continues term along with the sgn(s) with the aim of continuous improvement of control signal.
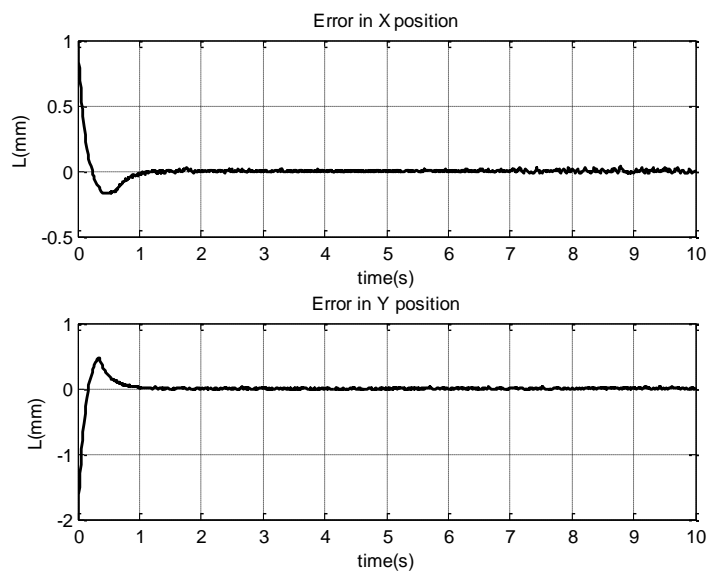

Fig 2. Tracking errors in the direction of $\mathrm{x}$ and $\mathrm{y}$ in Example 1

Fig. 2 shows the trajectory of probe along with the desired trajectory. This Figure confirms that in the presence of uncertainty and disturbances, the probe tracks the desired trajectory well.



Fig 3. Probe trajectory and desired trajectory in Example 1

Example 2: Consider the following desired path for the probe [38]

$\mathrm{x}_{\text {des }}=\mathrm{t}$
$\mathrm{y}_{\text {des }}=\frac{\mathrm{a}}{2}\left(1-\cos \left(\frac{\pi \mathrm{t}}{\mathrm{l}}\right)\right), \mathrm{a}=20, \mathrm{l}=30$

Suppose that the probe is subjected to uncertainty similar to the previous example. The difference is that there is no information on the upper bound of this uncertainty. So, the robust-adaptive controllers in Theorems 4-6 are used for this example. 
Consider the controller parameters as follow (the parameters are same for all of controllers)

$$
\Lambda=5 \cdot r=3 \cdot \rho=2 \cdot K=3
$$

Considering the probe parameters as in (47), the simulation results have shown in Fig. 3, Fig. 4 and Fig. 5with initial condition $\left[\mathrm{x}_{0}, \mathrm{y}_{0}\right]^{\mathrm{T}}=[1,-2]^{\mathrm{T}}$.


Fig. 4 tracking errors in the direction of $\mathrm{x}$ and $\mathrm{y}$ in Example 2

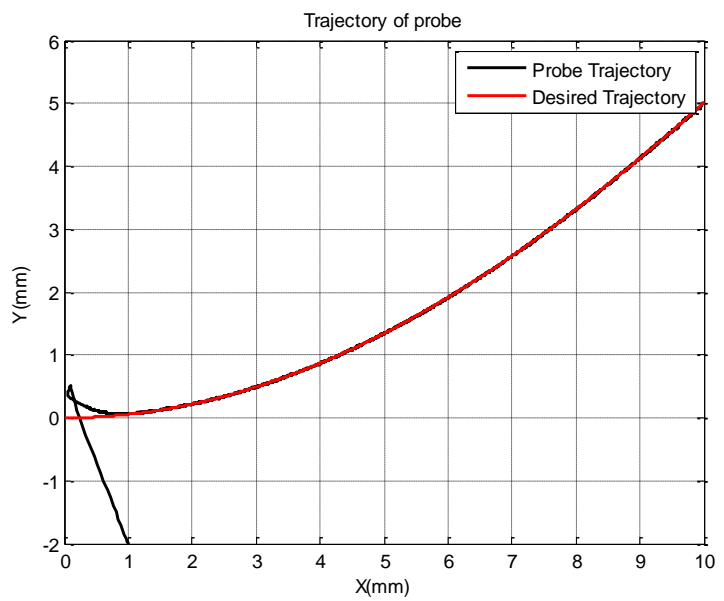

Fig. 5 Probe trajectory and desired trajectory in Example 2 

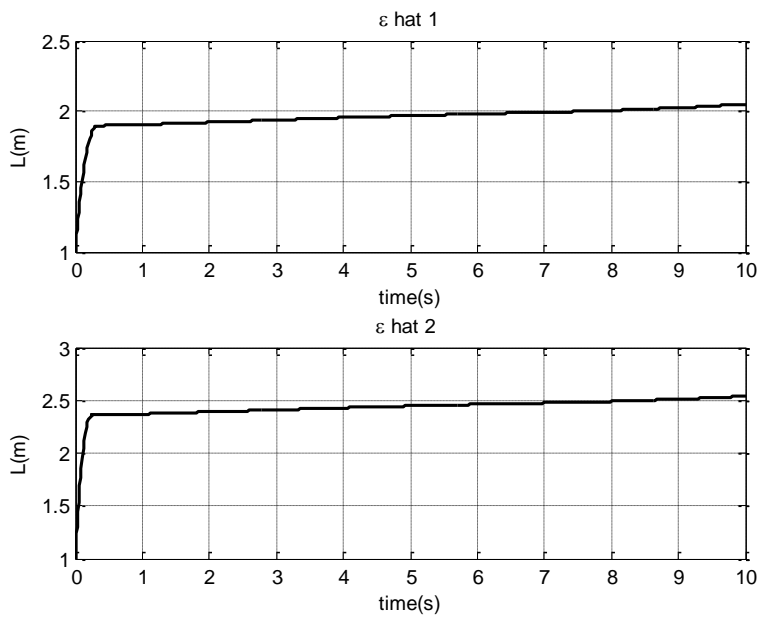

Fig. 6 Estimated parameters $\bar{\varepsilon}_{1}$ and $\bar{\varepsilon}_{2}$ in Example 2

Fig. 3 shows the trajectory errors that both go to zero. The trajectory of probe and desired trajectory both have been shown in Fig. 4. It is shown in this figure that the probe tracks the desired trajectory well. On the other hand, the estimations of the upper bound of disturbances have been shown in Fig. 5. This figure confirms that these estimations both are bounded.

\section{Conclusions}

The trajectory tracking problem of bio-inspired flexible probesin the presence of uncertainty and disturbance was dealt with. First, a robust sliding mode controller is established to deal with the uncertainties and output disturbances with this assumption that the upper band of uncertainty in the system is known. Then, an adaptive rule has been designed to estimate the upper bound of the uncertainty and disturbance and the sliding mode controller has been extended so that even if there is no information on the uncertainty, the system is still stable and the probe continues to tracks the desired trajectory. Simulation results demonstrated the effectiveness of the proposed method.

\section{References}

[1] N. Abolhassani, R. Patel, M. Moallem, "Needle insertion into soft tissue", e: A survey, Medical Engineering \& Physics 29, (2007) 413-431.

[2] B. Davies, "Medical robotics a bright future", The Lancet 368 (2006) S53-S54.

[3] G. Dogangil, B. L. Davies, F. Rodriguez Baena, "A review of medical robotics for minimally invasive soft tissue surgery", Proceedings of the Institution of Mechanical, (2008)

[4] G. Fichtinger, T. DeWeese, A. Patriciu et. al., "System for robotically assisted prostate biopsy and therapy with intraoperative CT guidance", Acad Radiol, 9(1), (2004), pp. 60-74.

[5] R. Alterovitz, J. Pouliot, R. Taschereau, I. Hsu, K. Goldberg, "Simulating needle insertion and radioactive seed implantation for prostate brachytherapy", Stud. in Health Tech. and Informat., (2003), pp. 19-25.

[6] N. Abolhassani, R. Patel, M. Moallem, "Needle insertion into soft tissue: A survey", Med. Eng. and Phys., 29(4), (2009), pp. 413-431. 
[7] R. Webster, J. Kim, N. Cowan, G. Chirikjian, A. Okamura, "Nonholonomic modeling of needle steering", The Intl. J. of Rob. Res, 25(5-6), (2006), p. 509.

[8] S. Misra, K. Reed, A. Douglas, K. Ramesh, A. Okamura, "Needletissue interaction forces for bevel-tip steerable needles", in Proc. IEEE Int. Conf. on Biomed. Rob. and Biomechat., (2008), pp. 224-231.

[9] S. P. DiMaio S. E. Salcudean, "Needle insertion modeling and simulation", IEEE Trans. on Rob. and Auto., 19(5),(2005), pp. 864-875.

[10] C. Duriez, C. Gu'ebert, M. Marchal, S. Cotin, L. Grisoni, "Interactive simulation of flexible needle insertions based on constraint models", in Proc. of MICCAI, (2009), pp. 291-299.

[11] R. Alterovitz, K. Goldberg, A. Okamura, "Planning for steerable bevel-tip needle insertion through $2 d$ soft tissue with obstacles", in Proc. of ICRA, (2007), pp. 1640-1645.

[12] S. P. DiMaio, S. E. Salcudean, "Needle steering and model-based trajectory planning", MICCAI, (2006), pp. 33-40.

[13] V. Duindam, R. Alterovitz, S. Sastry, K. Goldberg, "Screw-based motion planning for bevel-tip flexible needles in 3d environments with obstacles", ICRA, (2009), pp. 2483-2488.

[14] D. Glozman, M. Shoham, "Flexible needle steering and optimal trajectory planning for percutaneous therapies", MICCAI, (2004), pp. 137-144.

[15] L. Frasson, S.Y. Ko, A. Turner, T. Parittotokkaporn, J.F. Vincent, F. Rodriguez y Baena, STING: "a soft-tissue intervention and neurosurgical guide to access deep brain lesions through curved trajectories", Proceedings of the Institution of Mechanical Engineers-Part $\mathrm{H}$ : Journal of Engineering in Medicine 224 (2010), pp. 775-788.

[16] S.Y. Ko, L. Frasson, B.L. Davies, F. Rodriguez y Baena, "Software \& hardware integration of a biomimetic flexible probe within the ROBOCAST neurosurgical robotic suite", Hamlyn Symposium on Medical Robotics, London, UK, (2010).

[17] G. Fichtinger, T. DeWeese, and A. Patriciu et. al., "System forrobotically assisted prostate biopsy and therapy with intraoperativeCT guidance", Acad Radiol, vol. 9, no. 1 (2002), pp. 6074.

[18] N. Abolhassani, R. Patel, M. Moallem, "Needle insertion into softtissue: A survey", Med. Eng. and Phys., vol. 29, no. 4, (2007), pp. 413-431.

[19] D. Glozman M. Shoham, "Flexible needle steering and optimal trajectory planning for percutaneous therapies", in Proc. of MICCAI, (2004), pp. 137-144.

[20] N. Abolhassani, R. Patel, F. Ayazi, "Minimization of needle deflection in robot-assisted percutaneous therapy", Int. J. of Med.Robot. and Comp. Assis. Surg., vol. 3, no. 2, (2007) pp. 140-148.

[21] S. Misra, K. Reed, A. Douglas, K. Ramesh, A. Okamura, "Needletissue interaction forces for bevel-tip steerable needles", in Proc. IEEE Int. Conf. on Biomed. Rob. and Biomechat., (2008), pp. 224-231.

[22] S. P. DiMaio, S. E. Salcudean, "Needle insertion modeling and simulation", IEEE Trans. on Rob. and Auto., vol. 19, no. 5, (2003) pp. 864-875.

[23] C. Duriez, C. Guebert, M. Marchal, S. Cotin, L. Grisoni, "Interactive simulation of flexible needle insertions based on constraintmodels", in Proc. of MICCAI, (2009), pp. 291-299.

[24] R. Alterovitz, K. Goldberg, A. Okamura, "Planning for steerable bevel-tip needle insertion through $2 d$ soft tissue with obstacles", inProc. of ICRA, (2005), pp. 1640-1645.

[25] S. P. DiMaio, S. E. Salcudean, "Needle steering and model-based trajectory planning", in Proc. of MICCAI, (2003), pp. 33-40.

[26] V. Duindam, R. Alterovitz, S. Sastry, K. Goldberg, "Screw-based motion planning for bevel-tip flexible needles in $3 d$ environments with obstacles", Proc. of ICRA, (2008), pp. 2483-2488. 
[27] S.Y. Ko, B.L. Davies, F. Rodriguez y Baena, "Two-dimensional needle steering with a "programmable bevel"' inspired by nature: modeling preliminaries", IEEE/RSJ International Conference on Intelligent Robots and Systems Taipei, Taiwan, (2010), pp. 2319-2324.

[28] S.Y. Ko, L. Frasson, F. Rodriguez y Baena, "Closed-loop planar motion control of a steerable probe with a "programmable bevel" inspired by nature", IEEE Transactions on Robotics 27, (2011), pp. 970-983.

[29] R. J. Webster III, J. S. Kim, N. J. Cowan, G. S. Chirikjian, A. M. Okamura, "Nonholonomic Modeling of Needle Steering", The International Journal of Robotics Research, 25, (2007), pp. 509-525.

[30] R. Alterovitz, M. Branicky, K. Goldberg, "Motion Planning Under Uncertainty for Imageguided Medical Needle Steering", The International Journal of Robotics Research, 27, (2009), pp. 1361-1374.

[31] T. R. Wedlick A. M. Okamura, "Characterization of pre-curved needles for steering in tissue", in Proc. Annu. Int. Conf. IEEE Eng. Med. Biol.Soc., Sep. (2009), pp. 1200-1203.

[32] P. J. Swaney, J. Burgner, H. B. Gilbert, R. J. Webster, III, “A flexure based steerable needle: High curvature with reduced tissue damage”, IEEE Trans. Biomed. Eng., vol. 60, no. 4, (2013) pp. 906-909, Apr.

[33] S. Okazawa, R. Ebrahimi, J. Chuang, S. E. Salcudean, R. Rohling, "Hand-held steerable needle device", IEEE/ASME Trans. Mechatronics,vol. 10, no. 3, (2005) pp. 285-296, Jun.

[34] S. Y. Ko, L. Frasson, F. R. y Baena, "Closed-loop planar motion control of a steerable probe with a programmable bevel inspired by nature", IEEE Trans. Robot., vol. 27, no. 5, (2011), pp. 970-983, Oct.

[35] K. B. Reed, A. M. Okamura, N. J. Cowan, "Modeling and Control of Needles with Torsional Friction", IEEE Transactions on Biomedical Engineering, 56, (2009) pp. 2905-2916.

[36] D. Glozman, M. Shoham, "Flexible Needle Steering and Optimal Trajectory Planning for Percutaneous Therapies", Lecture Notes in Computer Science, 3217, (2005), pp. 137-144, 2004.

[37] D. Glozman and M. Shoham, "Image-Guided Robotic Flexible Needle Steering", IEEE transactions on robotics, 23, (2008), pp. 459-467.

[38] S. Young Ko and F. Rodriguez y Baena, "Trajectory following for a flexible probe with state/input constraints: An approach based on model predictive control", Robotics and Autonomous Systems, 60, (2012), pp. 509-521.

[39] D. S. Minhas, J. A. Engh, M. M. Fenske, C. N. Riviere, "Modeling of Needle Steering via DutyCycled Spinning", in the 29th Annual International Conference of the IEEE EMBS, Lyon, France, (2007), pp. 2756-2759.

[40] D. Minhas, J. A. Engh, C. N. Riviere, "Testing of Neurosurgical Needle Steering via DutyCycled Spinning in Brain Tissue in Vitro", in IEEE EMBS, Minneapolis, Minnesota, USA, (2009), pp. 258-261.

[41] N. A. Wood, K. Shahrour, M. C. Ost, C. N. Riviere, "Needle Steering System using Duty-Cycled Rotation for Percutaneous Kidney Access", in International Conference of the IEEE EMBS, Buenos Aires, Argentina, (2010), pp. 5432-5435.

[42] D. Glozman, M. Shoham, "Image-Guided Robotic Flexible Needle Steering", IEEE Transactions on Robotics, vol. 23, (2007), pp. 459-467. 